\title{
Comparing f-OFDM and OFDM Performance for MIMO Systems Considering a 5G Scenario
}

\author{
Felipe A. P. de Figueiredo ${ }^{\ddagger * \S}$, Nathália F. T. Aniceto ${ }^{\dagger \S}$, Jorge Seki ${ }^{\dagger}$ Ingrid Moerman* and Gustavo Fraidenraich ${ }^{\ddagger}$ \\ ${ }^{*}$ Ghent University - imec, IDLab, Department of Information Technology, Ghent, Belgium. \\ ${ }^{\dagger} \mathrm{CPqD}-$ Research and Development Center on Telecommunication, Brazil. \\ ${ }^{\ddagger}$ DECOM/FEEC-State University of Campinas (UNICAMP), Brazil. \\ Email: *[felipe.pereira, ingrid.moerman]@ugent.be, ${ }^{\dagger}\left[\right.$ nathalia, seki]@cpqd.com.br, ${ }^{\ddagger} \mathrm{gf} @$ decom.fee.unicamp.br \\ $\S$ Both authors contributed equally to this work.
}

\begin{abstract}
The advances mobile communications has seen in recent years has rendered the radio spectrum a limited and, hence, an expensive resource. Therefore, technologies that support unlicensed access to spectrum are needed. Therefore, the adoption of novel modulation schemes becomes of utmost importance to obtain better spectral-localization and reduce the OOBE (Out of Band Emission) inherent to OFDM (Orthogonal Frequency Division Multiplexing) and, consequently, mitigating the interference between secondary (unlicensed) and primary users. In this scenario, we access the gain in the bit error probability using f-OFDM in MIMO systems, both used in the 5G RANGE project.
\end{abstract}

Index Terms-5G RANGE, OFDM, f-OFDM, OOBE, Spectrallocalization, MIMO.

\section{INTRODUCTION}

In recent years, the growing demand for higher data rates has triggered an interest in new technologies that might be used to meet these new requirements [1]. In the current scenario, the expectation for $5 \mathrm{G}$ and everything it promises to offer is great. The future generation of mobile networks will present extremely challenging issues for telecommunications professionals. The new services defined by the 3GPP (3rd Generation Partnership Project) [2], seek to meet the following requirements:

- Ultra Reliable Low Latency Communications (URLLC): low latency communications and high reliability,

- Enhanced Mobile Broadband (eMBB): communications with higher data rate and spectral efficiency.

- massive Machine Type Communications (mMTC): massive communications between machines, with low complexity and power consumption.

In addition to these already proposed applications with their huge economic and social potentials, there are important services that are not being discussed by companies and other telecommunications organizations. With this perspective in mind, it was proposed by Brazilian and European institutions, a project with great challenges oriented to the $5 \mathrm{G}$ technology, which seeks to serve the needs of areas with low population density and geographical barriers. The purpose of the $5 \mathrm{G}$ RANGE is to implement mechanisms for the new network to provide flexible solutions that can offer connectivity in an economically viable way to rural urban areas. Brazil has an interest in serving regions because of the great importance of agribusiness in the Brazilian economy, which, due to connectivity problems, has difficulty in bringing new technologies to this sector.

One of the factors that prevented previous network generations from covering these regions was the high price of the spectrum with the use of licensed bands, making it impossible to invest in sparsely inhabited regions. In order to minimize this problem, $5 G R A N G E$ proposes the unlicensed allocation of TVWS (TV-White Spaces) in VHF (Very High Frequency) and UHF (High Frequency), and as a secondary user, significantly reducing network costs.

In this secondary user scenario, and also for improved spectrum utilization scenarios, it is of utmost importance the employ a physical layer waveform exhibiting low OOBE, providing spectrum agility and low interference to primary users. This requirement justifies the use of the f-OFDM waveform, which has its operation based on the filtering of the OFDM signal, reducing its OOBE. Taking into account the limitations of spectrum and the need for high data rates, we seek to increase the efficiency of the system through the use of MIMO (multiple input multiple output) technology and f-OFDM.

The aim of this article is to demonstrate the results obtained through simulations comparing OFDM techniques, used in LTE (Long Term Evolution), and f-OFDM, one of the potential modulations of the new generation, in MIMO systems. In OFDM systems, when signals are transmitted at adjacent frequencies (or channels), it is possible to observe that the signals leak into the adjacent channels. Therefore, by employing $\mathrm{f}-\mathrm{OFDM}$, the interference generated between the signals is expected to be smaller, decreasing the bit error rate, and increasing the spectral efficiency, as signals can better coexist. In addition, we also evaluate in this work the performance of several MIMO detectors such as Maximum Ratio Combining (MRC), Zero Forcing (ZF), Minimum Mean Squared Error (MMSE), Maximum Likelihood (ML) and Sphere Decoding (SD). A special attention is paid to this last decoder, as it presents performance similar to ML but with reduced complexity [4].

This paper is divided as follows: the section II presents the system model, presents a model for comparison of the performance of MIMO systems when OFDM and f-OFDM 


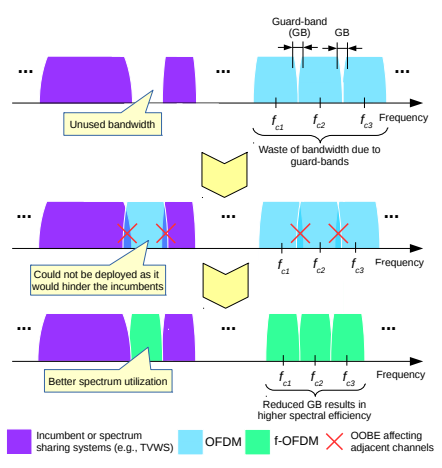

Figure 1. Signals at adjacent frequencies.

waveforms are used with several detectors, the results and discussion in section III, and the conclusions are presented in section IV.

\section{SyStem MODEL}

In this section, we present a description of the proposed study model, defining the employed modulation and demodulation methods and the detection algorithms evaluated at the receiving side of the system. We employ MIMO for transmission and reception in two scenarios, in the first one, each transmitted signal goes through OFDM modulation and the in second scenario, f-OFDM modulation is employed. $\mathrm{f}$ OFDM is one of the candidate methods for $5 \mathrm{G}$ waveforms and optimizes the operation of OFDM by reducing the OOBE. The model is based on the transmission three sets of four signals at three adjacent frequencies (i.e., $f_{c 1}, f_{c 2}, f_{c 3}$ ), as showed in Fig. 1, in both scenarios, so that it is possible to evaluate the effect of filtering on the OFDM signals. We consider $K=4$ single antenna devices simultaneously transmitting at each one of the three available frequencies and a BS (base station) equipped with $M$ antennas receiving and demodulating these signals.

Fig. 2 shows the uplink of a four-device/three-channel hypothetical MIMO system model, where each set of four devices, $K$, operates at frequencies $f_{c 1}, f_{c 2}$ and $f_{c 3}$, respectively. In addition, each device is equipped with one antenna, thus forming a MIMO MAC channel (Multiple Access Channel). In this work, each OFDM symbol is created by applying an 128point IFFT to the input signal, however, only 72 subcarriers are used for data transmission while the remaining ones are left for guard band. The subcarriers are spaced $15 \mathrm{KHz}$ apart, resulting in $72 \times 15 \mathrm{KHz}=1.08 \mathrm{MHz}$ of useful bandwidth. This signal is equivalent to a $1.4 \mathrm{MHz}$ LTE standard signal,

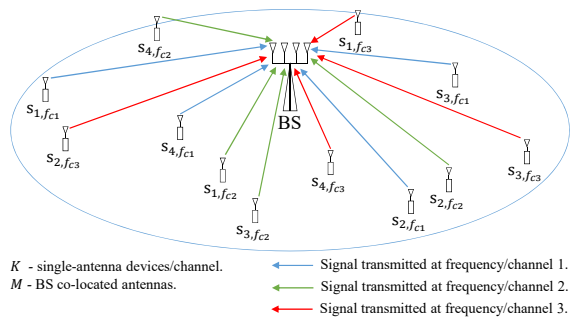

Figure 2. System model. where $1.08 \mathrm{MHz}$ is the useful band, and the rest is the guard band, which is used to reduce interference between adjacent channels. However, in our proposed study model, the distance in frequency between the end of a signal and the beginning of another is of only one subcarrier, i.e., $15 \mathrm{KHz}$.

\section{A. OFDM and f-OFDM Transceivers}

OFDM is a modulation technology used in many broadband communication systems [5]. Broadly speaking, it can be defined as an evolution of the FDM (Frequency Division Multiplexing) technique, [5, 6], with overlapping orthogonal subcarriers. With this feature, OFDM provides an increase in spectral efficiency level.

The transmission of an OFDM signal is done in parallel by means of different subcarriers, with the application of a simple modulation technique (e.g., QPSK, 16QAM, etc.) in each one of them. The modulated data bits are mapped into subcarriers, next the modulation symbols are processed by an IFFT (Inverse Fast Fourier Transform), resulting in samples in the time domain, after that, a CP (cyclic prefix), which consists of replicating the end of the OFDM symbol at the beginning of the signal block to be transmitted, is added to the signal, and then in the case of $\mathrm{f}-\mathrm{OFDM}$, the resulting OFDM signal is filtered. Finally, the OFDM or f-OFDM signal passes through a digital-to-analog conversion and then, is sent to the RF (radio frequency) front-end module, which transmits the signal at the desired frequency $f_{c i}, i=1,2$ and 3 .

In order to retrieve the original data sequence, the received signal passes through the following processing at the BS receiver side: RF and analog-to-digital conversion, optional filtering (only in case of f-OFDM), CP removal, FFT (Fast Fourier Transform) processing, subcarrier extraction, linear detection and demodulation.

In the case of f-OFDM modulation, filtering is performed after the IFFT block in order to reduce the OOBE, and thereby decrease the interference between adjacent signals. At the receiver side, as showed in Fig. 3, the filtering is performed after the analog-to-digital conversion. As can be seen in Fig. 3, the f-OFDM modulation/demodulation process is very similar to that of the traditional OFDM technique, differing only in the addition of low-pass filters [8, 9].

The ideal filter should be such that the transition band is as short as possible and with flat pass-band [7]. The ideal filter in frequency domain is given by the window function with its inverse given by the sync function as defined in Eq. (1).

$p(n)=\left\{\begin{array}{cl}\sin \left(\frac{\pi\left[12 \mathrm{NPRB}+2 \mathrm{~N}_{e}\right] n}{\mathrm{NFFT}}\right) / \frac{\pi\left[12 \mathrm{NPRB}+2 \mathrm{~N}_{e}\right] n}{\mathrm{NFFT}}, & n \neq 0 \\ 1, & n=0 .\end{array}\right.$

where NPRB is the number of physical resource blocks, NFFT is the FFT length used in the OFDM modulation, $L$ is the filter length and $-\frac{(L-1)}{2} \leq n \leq \frac{(L-1)}{2}$, and $\mathrm{N}_{e}$ is the excess bandwidth in number of subcarriers. The excess bandwidth is used to extend the flat region of the filter so that the subcarriers at the left and right borders of the OFDM symbols suffer less with attenuation.

However, it is impossible to implement a filter with such response, once it would require an infinite number of taps. 


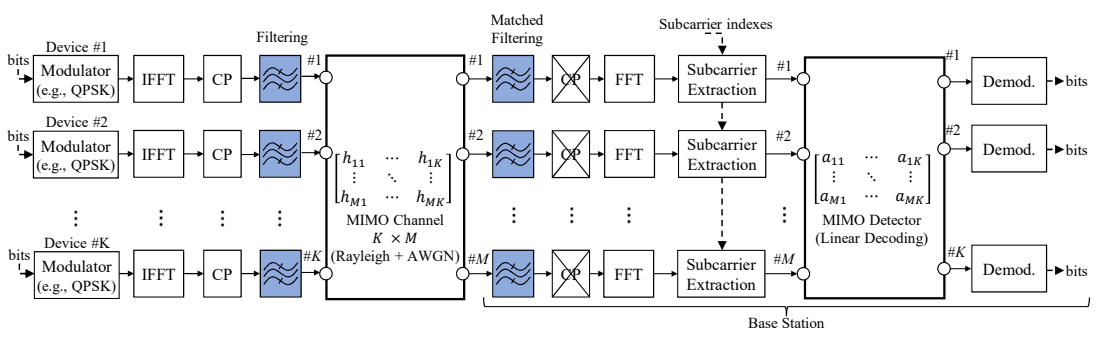

Figure 3. Block diagram of a MIMO uplink with OFDM/f-OFDM modulations. A cluster of $K$ devices seen at the transmit side share the same time-frequency resources, while the $\mathrm{BS}$, at the receiving side, is equipped with an array of $M$ antennas.

Therefore, in order to obtain feasible filters, we truncate the sync's response by applying a window, $w(n)$, which is defined next.

$$
w(n)=\left\{\frac{1}{2}\left[1+\cos \left(\frac{2 \pi n}{L-1}\right)\right]\right\}^{0.6}
$$

Finally, the normalized filter's coefficients are obtained as defined in Eq. (3),

$$
f(n)=\frac{p(n) \cdot w(n)}{\sum_{k} p(k) \cdot w(k)} .
$$

The appropriate filter design should maintain ICI (Inter Carrier Interference) and ISI (Inter Symbol interference) interference at acceptable levels [8].

As we will see later, in the f-OFDM case, the filter length does not need to be smaller than the CP length. This is due to the fact that the kind of filter we employ has the majority of its energy concentrated in the main lobe of the Sinc signal, which has length smaller than the CP length. Therefore, this allows filters with lengths longer than the $\mathrm{CP}$ one. Therefore, the energy leaking into the subsequent OFDM symbol's CP is very small and happens only for a short time [8].

\section{B. Detection Methods}

MIMO is a technology that has been used by the previous mobile communications networks [10,11], and will be widely applicable to 5G [2] and beyond networks. The received signal from $K$ single-antenna devices at a BS also equipped with $M$ antennas can be modeled according to Eq. (4),

$$
\mathbf{y}=\mathbf{H s}+\mathbf{n},
$$

where $\mathbf{s}$ is the $K \times 1$ transmitted signal vector, $\mathbf{y}$ is the $M \times$ 1 received signal vector, $\mathbf{H}$ is the $M \times K$ channel matrix and $\mathbf{n}$ is the $M \times 1$ Gaussian noise vector. As we employ a MIMO system for the transmission of several signals over the same time-frequency resource, the estimated signal can be determined by using one of the techniques described below. In this work we consider full channel knowledge.

1) Maximum Ratio Combining (MRC): In this method, the signals from each antenna are summed, and the signal branch is weighted by a factor proportional to its power level [13]. In this way, the branches with stronger signal are amplified, while the ones with lower signal are attenuated. A BS employing MRC detection aims at maximizing the received signal-tonoise ratio (SNR) of each device, neglecting the effects of multi-user interference (i.e., cross-talk). On one hand it has low implementation complexity, however, on the other hand, it presents poor performance in interference-limited scenarios, once it ignores multi-user interference. The MRC detection is defined by the product between the Hermitian conjugate of the channel response, $\mathbf{H}$, and the received signal vector [14], according to Eq. (5).

$$
\hat{\mathbf{s}}=\mathbf{H}^{\mathbf{H}} \mathbf{y} .
$$

2) Zero Forcing $(Z F)$ : The $Z F$ detection technique is a suboptimal linear detection algorithm used in communications systems, which focuses on recovering the transmitted signal, $\mathbf{s}$, by mitigating the interference among devices (i.e., multiuser interference). The detection is achieved by applying the pseudo-inverse of the channel to the received signal, $\mathbf{y}$ [12]. The ZF solution is found through Eq. (6),

$$
\hat{\mathbf{s}}=\left(\mathbf{H}^{\mathbf{H}} \mathbf{H}\right)^{-\mathbf{1}} \mathbf{H}^{\mathbf{H}} \mathbf{y} \text {. }
$$

ZF detection minimizes the interference between devices, but fails to tackle the effects of noise. Due to that fact, it performs well in interference limited scenarios and poorly in noise-limited scenarios. When compared to the MRC detector, it presents higher complexity, as it involves the computation of the pseudo-inverse of the channel matrices.

3) Linear Minimum Mean Squared Error (LMMSE): LMMSE detection minimizes the Mean Squared Error (MSE) between the estimate $\mathbf{A}^{H} \mathbf{y}$ and the transmitted signal $\mathbf{s}$, where A is the MMSE detection matrix. The LMMSE detector mitigates device interference while also taking the noise effect into account. Therefore, LMMSE detection maximizes the received SINR (signal to interference plus noise ratio). Therefore, when compared to other sub-optimal detectors such as ZF, and MRC, MMSE is the one with the best detection performance. The LMMSE detection is found with (7).

$$
\hat{\mathbf{s}}=\left(\mathbf{H}^{\mathbf{H}} \mathbf{H}+\frac{\sigma_{\mathbf{n}}^{2}}{\sigma_{\mathbf{s}}^{2}} \mathbf{I}\right)^{-\mathbf{1}} \mathbf{H}^{\mathbf{H}} \mathbf{y} .
$$

4) Maximum Likelihood (ML): ML is the known to be optimal detection technique [14]. ML tests all values of $\mathbf{s}$ and chooses the one with the smallest Euclidean distance to the received signal $\mathbf{y}$, according to Eq. (8).

$$
\hat{s}=\underset{\mathbf{s} \in \zeta^{K}}{\operatorname{argmin}}\|\mathbf{y}-\mathbf{H s}\|^{2},
$$

where $\zeta$ is the finite alphabet of $s_{k}, k=1,2, \ldots, K$. The disadvantage of ML detection is that it has to search over the space of $|\zeta|^{K}$ vectors, where $|\zeta|$ is the cardinality of the set 


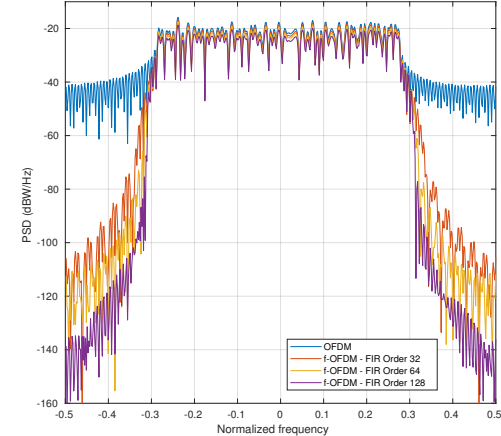

Figure 4. OFDM and f-OFDM PSD for filter orders 32, 64 and 128.

$\zeta$. Therefore, its complexity exponentially increases with the number of devices and modulation order.

5) Sphere Detector (SD): Although ML is an optimal detector, its use becomes not viable in practical systems due to its exhaustive search over all possible transmission vectors. On the other side, the SD, which was originally introduced by [15], appears in this scenario, and has its operation based on ML detector. Differently from ML, SD only searches for vectors laying within the radius of a sphere with its center in the received vector $\mathbf{y}$. It is important that the SD detector estimates the radius and the points that are inside the sphere, so as not to make the algorithm too complex. One interesting property showed by SD is that if it finds a valid vector, it will be the exact same vector the ML detector would find in that case.

\section{RESUlTS AND Discussion}

In this section we present and assess the results regarding the two model scenarios using MIMO systems. We evaluate the performance gain obtained with the use of f-OFDM over OFDM, by means of experimental results. We employ the system model depicted in Fig. 2 and focus on the detection performance of the signals transmitted at the central frequency.

In Fig. 4 the comparison between the power spectral density (PSD) of OFDM and f-OFDM signals is presented. As can be noted, the addition of the FIR filter to the OFDM transmission chain drastically reduces OOBE. This reduction ranges from $-40 \mathrm{dBW} / \mathrm{Hz}$ with OFDM to $-100 \mathrm{dBW} / \mathrm{Hz},-110 \mathrm{dBW} / \mathrm{Hz}$ and $-120 \mathrm{dBW} / \mathrm{Hz}$ at a frequency of $0.4 \times f s$ (where $f s$ is the sampling rate) with FIR filters of orders 32, 64 and 128, respectively.

Fig. 5 shows the base-band impulse response of the designed filter with bandwidth equal to $72 \times 15 \mathrm{KHz}+2 \times \mathrm{N}_{e}$. It can be noticed that the main energy of the filters is confined within the the sinc's main lobe, which in this case, spans 2.084 $[\mu s]$. Therefore, the filter's energy stays confined within the $\mathrm{CP}$ length (for normal $\mathrm{CP}$ it is approximately $4.7[\mu s]$ ), and consequently, ISI stays within tolerable levels.

In Fig. 6 we present the frequency responses of the designed filters for f-OFDM with NPRB $=6, \mathrm{~N}_{e}=3$, and filter order 32, 64 and 128 respectively. The figure shows the 3 $\mathrm{dB}$ cutoff frequency (red-dashed lines) of the filters, which, as designed, happens at half of the useful bandwidth plus the excess bandwidth, $\mathrm{N}_{e}$, i.e., $1.08 \mathrm{MHz} / 2+3 \times 15 \mathrm{KHz}$ $=585 \mathrm{KHz}$. As expected, the 128-th order filter presents a steeper transition region, which results in less interference to adjacent channels and a better frequency-localization when compared with OFDM. The figure also shows the frequency (green-dashed lines) of the subcarrier at the edge of the OFDM symbol. As can be seen, as the filter order increases, the subcarriers at the edges of the symbol are less affected by attenuation/overshoot at the edges.

In Fig. 7 we present the BER evaluation when QPSK modulation is used with $10^{7}$ Monte Carlo iterations. The BER measurements we present are an average over all the subcarriers carrying data. As can be seen, the BER is lower when using f-OFDM modulation for all the considered filter orders. For the sake of performance comparison, we add to the figure the Matched Filter Bound (MFB) as benchmark for the BER comparisons. The MFB is also called in the literature as the perfect interference-cancellation bound. As it is suggested by its name, the MFB performs as the $k$ th device of a matched-filter receiver in the absence of other sources of interference such as devices at adjacent channels and multiuser interference, i.e., cross-talking interference caused by devices using the time-frequency resources. As noticed, the BER of f-OFDM modulation improves as the filter order increases. Additionally, we also see that f-OFDM with SD and ML detection approaches the MFB faster as the filter order increases.

As expected, MMSE detection has the best performance and MRC detection has the worst one among the studied detectors. Considering a BER of $10^{-2}$ and taking the MMSE detector's performance into account we see that there is a gain of $\approx 1[\mathrm{~dB}], \approx 2[\mathrm{~dB}]$, and $>3.5[\mathrm{~dB}]$ for filter orders 32,64 and 128 respectively. Additionally, it is important to emphasize the use of SD detection, which has smaller computational complexity when compared to the ML detection and still has performance similar to that detector.

It is also important to notice that for a filter order of 32 (see Fig. 7 (a)) and $\mathrm{N}_{e}=3$ the BER for $\mathrm{SD}$ and ML detectors reaches a BER floor of approximately $10^{-4}$ for SNR greater than $12[\mathrm{~dB}]$. From that point onward the performance of $\mathrm{f}-\mathrm{OFDM}$ is worse than that of OFDM with SD and ML detection. This is due to the fact that the subcarriers at the edges of the OFDM symbols are heavily affected by

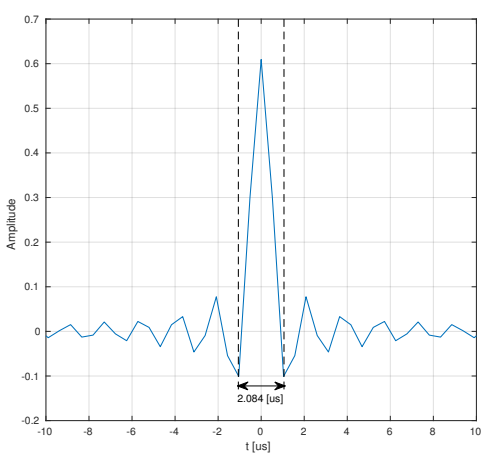

Figure 5. Impulse response of the designed filter for f-OFDM with bandwidth equal to $72 \times 15 \mathrm{KHz}+2 \times \mathrm{N}_{e}$. 


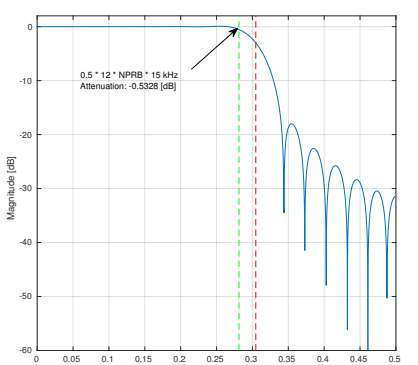

(a) FIR order 32.

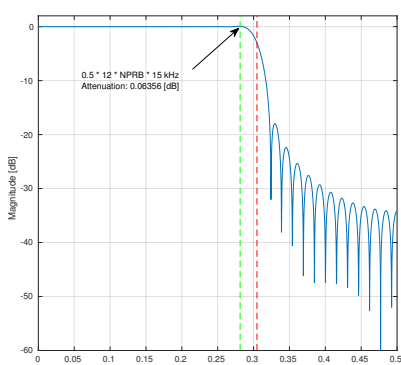

(b) FIR Order 64.

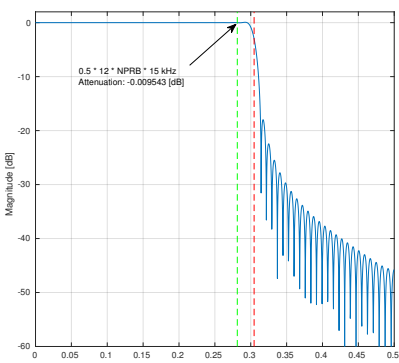

(c) FIR order 128.

Figure 6. Frequency responses of the designed filters for f-OFDM with NPRB $=6, \mathrm{~N}_{e}=3$, and filter orders 32 (a), 64 (b), 128 (c).

the filter's poor performance at its edges (i.e., attenuation and overshoot) becomes noticeable as can be seen in Fig. 6 (a). The attenuation and overshoot at the edges of the filter response are the limiting factor for BER at high SNR values. In order to validate this assumption, we also show in Fig. 7 (a) BER results for excess bandwidths, $\mathrm{N}_{e}$, of 3 and 10 excess subcarriers. That increases the flat region of the pass-band, making the filter flatter at the edges, and consequently, the BER for f-OFDM with SD and ML detectors do not present a floor value anymore. This behaviour is also showed with higher order filters (e.g., 64 and 128), were we do not verify the floor effect, once they exhibit a longer flat region (as is seen in Fig. 6 (b) and (c)).

Another interesting results is depicted in Fig. 8. In this figure we see the benefits of having a BS equipped with a larger number of antennas. As can be seen, as the number of antennas increases the BER performance of the detectors asymptotically approaches that of the OFDM MFB. As can also be noticed, the performance of the sub-optimal detectors, $\mathrm{ZF}$ and MMSE, asymptotically approaches the performance of the almost-optimal and optimal detectors, SD and ML. This is due to the fact that as the number of antennas increases, the interference and noise tend to vanish as the devices' channels become asymptotically orthogonal due to the law of large numbers [16]. These results clearly prove that the interference caused by users transmitting at closely separated adjacent channels can be mitigated by having a BS equipped with a large number of antennas.

It is known that the OFDM modulation exhibits high Peakto-Average Power Ratio (PAPR), which is a limiting factor in some cases. Due to its nature, OFDM modulation presents large peak variations in time domain, and as a consequence, it has higher PAPR than single-carrier modulations. Therefore, this effect poses a challenge to the RF power amplifier design and degrades the efficiency of the power amplifier as it has to back off, i.e., as the OFDM signal varies a lot, the operational point of the amplifier has to be reduced so that high signal values do not get into the amplifier's non-linear region. A signal is distorted when it gets into the amplifier's nonlinear region. This distortion causes inter-modulation among subcarriers and creates OOBE, which is a effect we want to avoid. Additionally, low PAPR is a highly desirable feature in device-to-device (D2D) and vehicle-to-anything (V2X) communications.

In Fig. 9 we assess the PAPR presented by single carrier,
OFDM and f-OFDM modulation schemes. In the figure we measure the Complementary Cumulative Distribution Function (CCDF) for each one of the compared modulations. The CCDF gives the probability of the instantaneous power of a signal be greater than a specified level over its average. We observe that the probability of the power of the OFDM and f-OFDM modulated signals being more than $3[\mathrm{~dB}]$ above its average power level is higher than for a QAM modulated signal. For example, the power level above the average power level is of $2.46[\mathrm{~dB}], 3.62[\mathrm{~dB}], 4.11[\mathrm{~dB}], 4.49[\mathrm{~dB}]$ and $5.02[\mathrm{~dB}]$ with a percentage of $10 \%$ for QAM, OFDM, f-OFDM 32, f-OFDM 64 and f-OFDM 128 respectively. We conclude that, although reducing the OOBE, the $\mathrm{f}-\mathrm{OFDM}$ modulation presents as a drawback, a PAPR that is higher than that presented by the OFDM modulation and that gets worse as the filter order increases.

\section{CONCLusions}

In this paper, we assessed the influence of the interference among adjacent signals in a MIMO system when OFDM and f-OFDM modulations are employed. The MIMO system performance was analyzed by means of the BER calculation, using MRC, ZF, MMSE, SD and SD detection methods. We demonstrate the importance of filtering the OFDM modulate signal as a way to mitigate interference at adjacent channels.

Our analysis concludes that f-OFDM equipped systems can perform better than OFDM, when power amplification is not an issue, i.e., the RF power amplifier is designed so that the high signal variation does not get into the amplifier's nonlinear region. Given that condition, we conclude that f-OFDM is an excellent candidate for future generations of wireless and mobile networks. Therefore, f-OFDM systems allow closer frequency coexistence of devices, which increases the spectral efficiency, as the distance among adjacent channels can be decreased.

As future work, we plan to study ways of having lower PAPR while still keeping the reduced OOBE (i.e., good spectral-localization) presented by f-OFDM modulation. One possible direction is the integration of f-OFDM with DFTspread OFDM.

\section{ACKNOWLEDGMENT}

This work was funded by (i) the European Union's Horizon 2020 research and innovation programme under grant agreement No. 732174 (ORCA project) and also by (ii) 


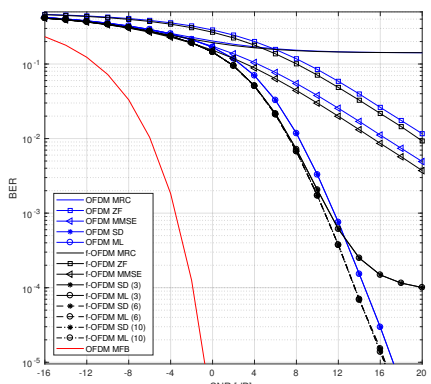

(a) FIR order 32.

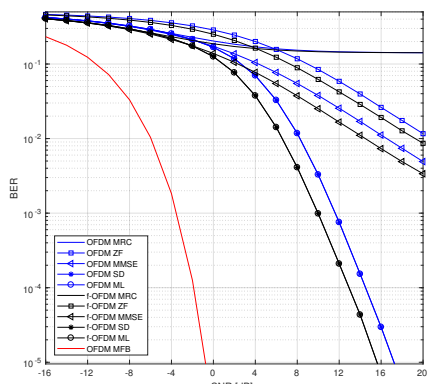

(b) FIR order 64 .

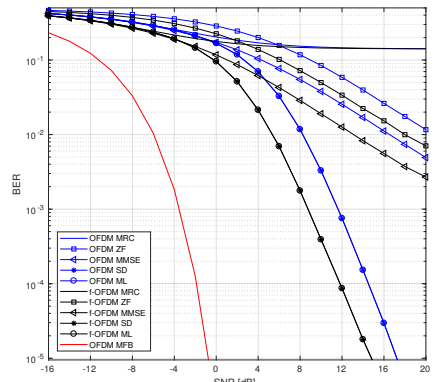

(c) FIR order 128.

Figure 7. BER performance for MIMO OFDM versus MIMO f-OFDM detection on uplink direction with $K=4, M=4, \mathrm{NPRB}=6, \mathrm{~N}_{e}=3$ and filter orders 32 (a), 64 (b), 128 (c).

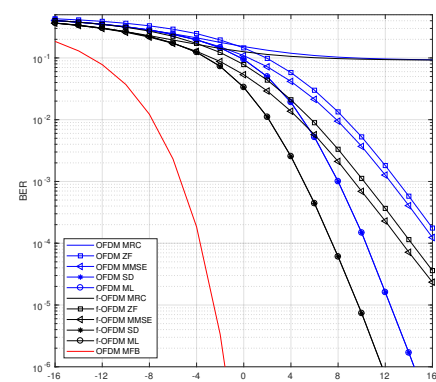

(a) $\stackrel{\text { SNe [da] }}{=} 6$.

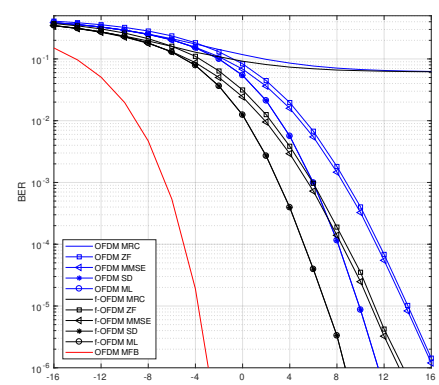

(b) $M \stackrel{\text { SNR [de] }}{=} 8$.

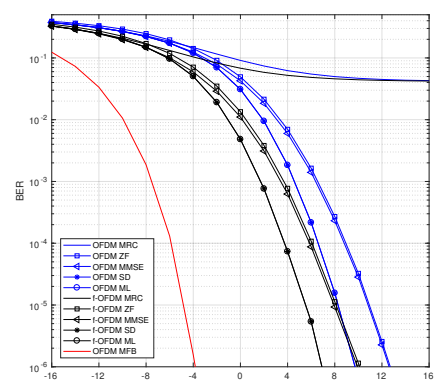

(c) $M \stackrel{\text { SNR [da] }}{=} 10$.

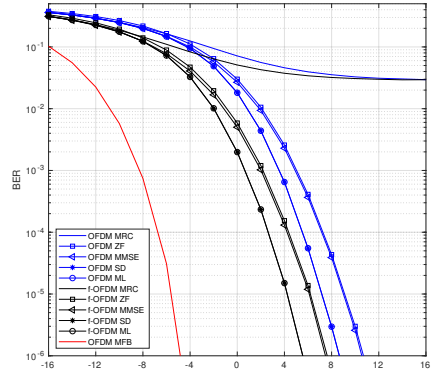

(d) $M \stackrel{\text { SNR[DB] }}{=} 12$.

Figure 8. BER performance for MIMO OFDM versus MIMO f-OFDM detection on uplink direction with $K=4$, NPRB $=6$, filter order $128, \mathrm{~N}_{e}=3$, and number of antennas, $M, 6$ (a), 8 (b), 10 (c), 12 (d).

the Center for Research and Development in Telecommunications $(\mathrm{CPqD})$, through participation in the project 5GRANGE (Technical Cooperation Agreement for Research and Development between RNP and CPqD - H2020-EUB-2017)

\section{REFERENCES}

[1] Mansoor Shafi, Andreas F. Molisch, Peter J. Smith, Thomas Haustein, Peiying Zhu, Prasan De Silva, Fredrik Tufvesson, Anass Benjebbour, and Gerhard Wunder, 5G: A Tutorial Overview of Standards, Trials, Challenges, Deployment, and Practice, IEEE Journal on Selected Areas in Communications, vol. 35, no. 6, pp. 1201-1221, June 2017.

[2] 3GPP, Technical enhancements and improvements for rel-15, 3rd Generation Partnership Project (3GPP), Technical Specification (TS) 21.915, Jun 2015

[3] $5 G$ RANGE, http://5g-range.eu/, 2017, [accessed on 01-May-2019].

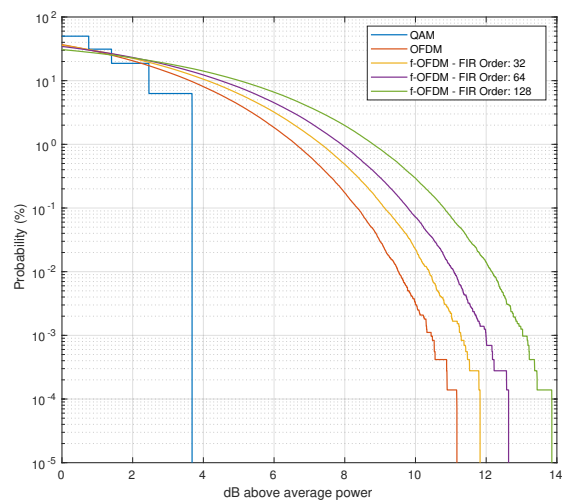

Figure 9. CCDF comparison of single-carrier and OFDM/f-OFDM modulation schemes.
[4] T. E. A. V. Agrell, E. and K. Zeger., Closest point search in lattices, IEEE Transactions on Information Theory, vol. 48, no. 8, pp. 2201-2214, Aug. 2002.

[5] S. B. Weinstein, Introduction to the history of OFDM, IEEE Commun. Mag., vol. 47, no. 11, pp. 26-35, 2009.

[6] Y. Li, J. H. Winters, and N. R. Sollenberger, MIMO-OFDM for wireless communications: Signal detection with enhanced channel estimation, IEEE Trans. Commun., vol. 50, no. 9, Sept. 2002.

[7] Felipe A. P. de Figueiredo, R. Mennes, X. Jiao, W. Liu, and I. Moerman, A spectrum sharing framework for intelligent next generation wireless networks, IEEE Access, vol. 6, pp. 60704-60735, Nov. 2018.

[8] L. Zhang, A. Ijaz, P. Xiao, M. M. Molu, and R. Tafazolli, Filtered OFDM systems, algorithms and performance analysis for $5 G$ and beyond, IEEE Trans. Commun., vol. 66, no. 3, pp. 1205-1218, Nov. 2017.

[9] J. Abdoli, M. Jia, and J. Ma, Filtered OFDM: A new waveform for future wireless systems, in 2015 IEEE 16th International Workshop on Signal Processing Advances in Wireless Communications (SPAWC), 2015.

[10] 3GPP, 3rd Generation Partnership Project; Technical Specification Group Radio Access Network; Evolved Universal Terrestrial Radio Access (EUTRA); Physical layer procedures (Release 8), 2008.

[11] 3GPP, LTE; Feasibility study for Further Advancements for E-UTRA (LTE-Advanced) (Release 9), TR 36.912, 2009.

[12] A. Klein, G. Kawas Kaleh, and P. Walter Baier, Zero forcing and minimum mean-square-error equalization for multiuser detection in code-division multiple-access channels, IEEE Trans. Veh. Technol., vol. 45, no. 2, pp. 276-287, May 1996.

[13] K. Tiwari and D. Saini, SER improvisation of mimo-mrc system over weibull-gamma fading channel, in 2015 International Conference on Signal Processing and Communication (ICSC), pp. 70-73, March 2015.

[14] I. F. Labaran, Spatial modulation: A comparison of maximum receiver ratio combining and maximum likelihood detectors, in 2014 11th International Conference on Electronics, Computer and Computation (ICECCO), pp. 1-3, Sep. 2014.

[15] U. Fincke, and M. Pohst, Methods for calculating vectors of short length in a lattice, including a complexity analysis, Mathematics Subject Classification, vol. 44, pp. 463-471, 041985.

[16] Felipe A. P. de Figueiredo, Fabbryccio A. C. M. Cardoso, Ingrid Moerman, Gustavo Fraidenraich, On the Application of Massive MIMO Systems to Machine Type Communications, IEEE Access, vol. 7, no. 12, pp. 2589-2611, December 2018. 